\title{
QUALITY OF SERVICE IMPROVEMENTS IN IEEE 802.11AX WI-FI
}

\author{
B.T. Vijay and B. Malarkodi \\ Department of Electronics and Communication Engineering, National Institute of Technology, Tiruchirappalli, India
}

\begin{abstract}
IEEE 802.11ax is the latest high rate Wi-Fi technology (also known as high efficiency Wireless) introduces some new features like OFDMA, Uplink multi-user MIMO (UL MU-MIMO) to deliver benefits in reliability, capacity, and speed. 802.11ax is better suited to some new use cases, such as live video streaming and IoT than previous versions of $\mathrm{Wi}-\mathrm{Fi}(802.11 \mathrm{n} / \mathrm{ac})$. In this article, we study the Quality of Service (QoS) mechanism to guarantee certain levels of service to various traffic flows with the help of weighted fair queuing (WFQ) hierarchical scheduler. The multi-user mode of enhanced distributed channel access (MU mode EDCA) illustrates the controlling aspects, issues, and possible solutions of EDCA for the 802.11ax standard to satisfy QoS requirements in dense deployment scenarios.
\end{abstract}

Keywords:

Access Category (AC), Multi-User (MU), Enhanced Distributed Channel Access (EDCA), 802.11ax, Quality of Service (QoS), Weighted Fair Queuing (WFQ)

\section{INTRODUCTION}

Wi-Fi continues the most effective tool to deliver high-speed, high-performance connectivity at home, in the office, and in public places such as stadiums and shopping centers. However, to support increasing data demand and changing user requirements, a new generation of the technology is needed. IEEE 802.11ax (High Efficiency Wireless (HEW)) provides features to meet this need, including tools to boost capacity and reliability as well as an increase in average user speeds. 802.11ax named as the sixth generation of Wi-Fi technology and sees some crucial changes in the designed when compared to 802.11ac [1] [8].

The IEEE 802.11 ac will face challenges supporting new social experiences alongside the expected growth of the Internet of Things (IoT) applications. IoT is characterized by a ballooning number of devices in the home, workplace and public places, which send small amounts of data to the cloud regularly. This needs wireless networks designed to support short bursts of data from many devices [5]. Since the IoT applications usually have diverse requirements, the internet should also make a distinction between traffic flows and their demands to ensure each flow will get its desired level service. In general, Quality of service (QoS) can be explained as the ability of the network to ensure certain levels of service to various traffic flows [1] [4] [7]. The distributed coordination function (DCF) scheme not fulfill the QoS demands for applications like video conferencing because it's not in dynamic nature contention window maximum aspects. The adaptation of OFDMA to make the most efficient use of spectrum and MU mode EDCA for equal sharing to all Wi-Fi users with the same network configuration. Multi-User downlink (MU DL) is defined for MU-MIMO procedure in 802.1 lac for sharing a TXOP channel access scheme for MU-MIMO. Channel Access follows the rules for the Access Category of primary AC and transmission success is judged by primary transmission only. We could have two options of DL MU, (1) Using sharing a TXOP method for both DLMU-MIMO and DLOFDMA, and (2) Using virtual DL MU queue like virtual UL_MU queue. Opt.1 is simple, but it has some regulations since the failure of primary transmission is regarded as the transmission failure, an inefficient procedure should be taken, i.e., CW should be doubled. Multi-TID cannot be used for primary transmission [11]. Opt.2 requires the new procedure, but it could be more flexible, transmission success can be more appropriately defined (e.g., only one response of DL MU transmission can be considered as a success). Multi-TID A-MPDU could be utilized for DL MU as well [9] [11] [12].

In many cases, the capacity of an 802.11ax network improves by a factor of six compared with 802.11 ac enabling and futureproofing the Wi-Fi network for IoT. OFDMA in the uplink (ULOFDMA) is also the key to the increase in data upload requirements, such as for live video streaming applications. Use of scheduling in UL-OFDMA delivers a smoother, low-latency experience to multiple users at the same time. While UL-OFDMA helps with a low-latency uplink experience when many users are on the network, the benefits of UL MU-MIMO are better experienced with a high volume of data from a smaller group of users for example in the home. The MU mode EDCA is an extension of the EDCA presented in the 802.11e amendment to support prioritized quality of service (QoS) for high efficiency stations (HE STAs). HEW MU UL channel access has not defined yet but many comments are related to AC, and channel access of UL MU transmission discussed in study group 802.11ax. In this article we mainly look at EDCA modifications for 802.11ax in that briefly explained distributed fair scheduling (DFS) [7] scheme in section 4 also we simulated WFQ algorithm with the help of four type of service (ToS) classes also known as intra access categories prioritization (802.11aa) [14] parameters to meet stringent QoS requirements for dedicated users. Lately, we investigate the MU mode EDCA functionalities for both uplink (channel access for trigger frame) and downlink cases, here buffer status report (BSR) procedure is adopted in 802.11ax for uplink MU transmission. MU mode EDCA control and its issues for new sets of parameters which are too utilized after trigger frame reception are noted.

\section{BACKGROUND}

With the development of throughput in Wi-Fi, many new kinds of data packets will flow through Wi-Fi networks. This article discusses the EDCA enhancement for multiple access (MU MIMO/OFDMA) with the help of differentiable CSMA/CA parameters adjustment like dynamic nature in contention window $\left(C W_{\max }\right)$ and QoS provisioning in 802.11ax with the help of distributed fair scheduling for betterment of network throughput. Hierarchical scheduling model to deal with new situations using centralized weighted fair queuing (WFQ) scheme. HEW address 
the parameterized and guaranteed quality of experience (QoE) may be a solution to improving real-world performance.

At present day dense scenarios only, the IEEE 802.11e/ae/aa standard is available for QoS enhancements for Wi-Fi through modifications to the MAC layer. In 802.11e have two different variations, HCCA mechanism for parameterized QoS, and EDCA mechanism for differentiated QoS. IEEE 802.11e EDCA defines traffic classes to analyze packets of four Access Categories.

$\mathrm{AC} \_\mathrm{VO}$ is the highest priority for voice applications, AC_VI is the next highest priority for video applications, $\mathrm{AC}$ BE is the third priority for best-effort data, and $\mathrm{AC} \mathrm{BK}$ is the lowest priority for background data. Similarly, 802.11 ae defines a QoS management frame (QMF), only QoS stations can implement QMF policy. A QMF station uses access category AC_VO to transmit management frames to STAs that do not support the QMF service. Lastly, IEEE 802.11aa define to help robust streaming of audio-video transport streams for PHY/MAC layer [6] [14]. Stream classification service (SCS) offers advanced differentiation idea of 802.11e EDCA access mechanism, an Intra-access category prioritization to improve a granularity for the service differentiation further. A couple of additional queues are included in the EDCA access to assist the AV streaming, $\mathrm{AAC} \mathrm{VO}$ for voice and for video AAC VI, which explained in upcoming section in terms of 4 type of service (ToS) classes based implemented in WFQ mechanism.

The packets for the same AC are enqueued in FIFO queue with drop tail technique (EDCAF). In the HEW system data traffic, except big data, there are still many short packets. They may be the minor part of total data volume, but a significant amount of data packets [3] for example, while $4 \mathrm{~K}$ and $8 \mathrm{~K} / \mathrm{Ultra}-\mathrm{HD}$ supported, there are also many $360 \mathrm{P} / 480 \mathrm{P}$ videos, and some users are extreme downloading, others may check their e-mail or Facebook. The original EDCA does not distinguish detail property of the data packets in the same AC. So, it may be bad for the short packets.

\section{IEEE 802.11AX}

In this section, we discussed why IEEE 802.11 ax standard is the 6th generation WiFi. The 802.11ax amendment also called as high efficiency Wireless (HEW) because of its improving capacity targeted up to $10 \mathrm{x}$ but present situation we can visualize up to $4 \times$; it works on both the bands such as $2.4 \mathrm{GHz}$ and $5 \mathrm{GHz}$ to improve spectral efficiency in highly congested APs/ dense environments. The following components give us clear justification like 802.11ax is a successor 802.11ac [8] [10].

- UL/DL OFDMA

- UL/DL 8x8 MU-MIMO

- OFDMA long symbol duration

- New PPDU formats

- BSS coloring

- TWT power saving

Orthogonal Frequency Division Multiple Access (OFDMA): multi-user version of OFDM enabling concurrent AP communication (Uplink/Downlink) with multiple clients by assigning subsets of subcarriers, called Resource Units (RUs) to the individual clients. Based on client traffic needs, the AP can allocate the whole channel to only one user or may partition it to serve multiple users simultaneously.

Multi-User Multiple Input Multiple Output (MU-MIMO): Introduced in very high throughput Wi-Fi (802.11ac), MUMIMO technology allows the simultaneous transmitting of multiple frames to different receivers at the same time on the same channel using multiple RF streams to provide greater efficiency. $11 \mathrm{ax}$ adds $8 \times 8$ and uplink MU-MIMO services to provide significantly higher data throughput.

$Q A M$ - 1024: Modulation techniques are used to optimize throughput and range. The number of points in the modulation constellation determines the number of bits conveyed with each symbol. 802.11ac uses 256 QAM which transfers 8 bits/symbol. 802.11ax supports 1024 QAM, using 10 bits/symbol for a $25 \%$ increase in throughput.

Long Symbol Duration: 4× larger OFDM symbol times increase efficiency and improves robustness, especially for transmission in outdoor scenarios.

Preamble Updates or PPDU formats: Modified frame formats provide High Efficiency (HE) and legacy information to support new advanced capabilities as well as information required to support legacy stations and backward compatibility.

Overlapping Basic Service Set (OBSS): To improve spatial reuse efficiency and performance, 11 ax adjusts the carrier sense operation based on the color of the BSS. Depending on the BSS the traffic is generated from, the station can use different sensitivity thresholds to transmit or defer. This results in higher overall performance.

Target Wake Time (TWT): TWT allows the AP to schedule a series of times for a station to wake up at scheduled intervals to exchange data frames. This allows the station to sleep longer and reduces energy consumption. Key capability for IOT devices [10-11].

\section{DISTRIBUTED FAIR SCHEDULING}

In this section we reviewed the best way to provide QoS provisioning in Wi-Fi is distributed fair scheduling (DFS). The intention of fair scheduling could be defined by a flawless fluid flow type as outlined in Eq.(1). Within, $r_{i}$ refers to the bandwidth utilized by flow $i$ and $\phi_{i}$ refer to the weight of the flow; which is the service share of each flow need to be proportional to the flow weight through any period. It has been verified that fair scheduling gives end-to-end delay range for any well-behaved traffic flows [7].

$$
\frac{r_{i}}{\phi_{i}}=\frac{r_{j}}{\phi_{j}} \forall i, \forall j
$$

Wi-Fi fairness scheduling attempts to allocate bandwidth between flows owned by distributed nodes in the network, complying while using fairness goal in Eq.(1). The distribution of flows tends to make the design of a fair scheduling algorithm in Wi-Fi a demanding task. The purpose of a distributed setup of fair scheduling paradigm is to make the simultaneous mode of operation as a hierarchical scheduler could do. The service progress information may differ with schemes concerning the unique contents, such as the type of service (ToS), traffic sent/received/dropped and queuing delay. 


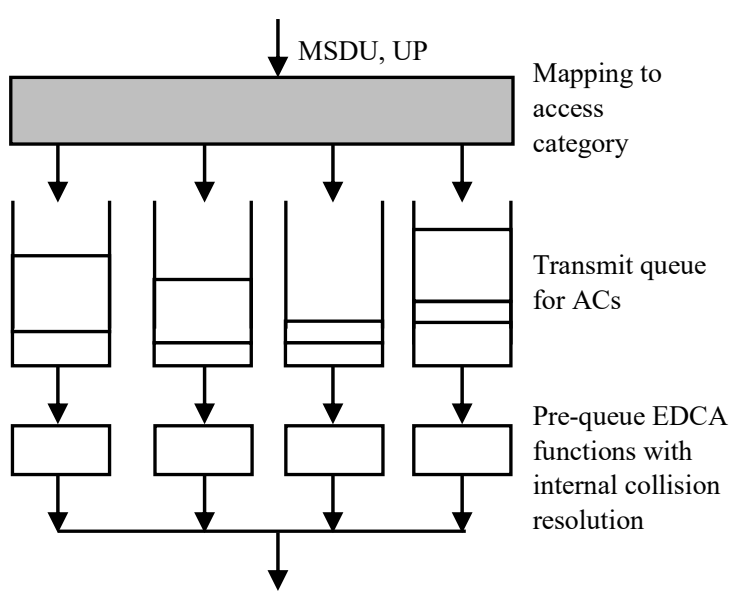

Fig.1(a). Former EDCA Reference Model

In the Hierarchical Scheduler, WFQ (Weighted Fair Queuing) is used for example is shown in Fig.1(b) [8]. When compared with original EDCA, the Hierarchical Scheduler can do better with the bursty short packets [7]. Weighted fair queuing is a centralized scheduling algorithm needs to know whether other flows are backlogged or not in real-time. The real-time flow backlog status will create a significant level of traffic load to the network to reduce such overhead, DFS decides to replicate the self-clocked fair queuing (SCFQ) scheduling model. SCFQ needs minimal status information sharing, which can be the finished tag of the last sent packet. The Hierarchical Scheduler may be more flexible according to the taxonomy of QoS based DCF extensions four categories. (1) Predetermined static differentiation, (2) distributed priority access, (3) distributed fair packet scheduling and (4) distributed reservation access. These four algorithms are implemented in hierarchical scheduler which is shown in Fig.1.

The Hierarchical Scheduler model might be considered in HEW, due to the diversity of packets. The detail parameter setting, and scheduler algorithm may be determined in the future. The enhancement of EDCA needs to be considered in future discussions to deal with new traffic models in HEW. Multi-user mode of EDCA or OFDMA based random access is future of channel access in 802.11ax. Queuing schemes offer estimated network service by giving specific bandwidth, handled jitter and latency, and much better packet loss attributes. The fundamental concept is to allocate resources, for example, processor and buffer usage space for sensitive data. The following schemes demand tailored configuration of output interface queues.

Priority Queuing $(P Q)$ makes sure that during congestion the top priority data won't get slow down by lower priority traffic. However, lower priority traffic can encounter considerable delays. PQ is made for situations that give attention to missioncritical data, not including or deferring less essential traffic during periods of congestion.

Custom Queuing (CQ) assigns a specific portion of the bandwidth to each queue to ensure expected throughput for other queues. It is made for situations that require ensuring an acceptable level of service to all traffic.

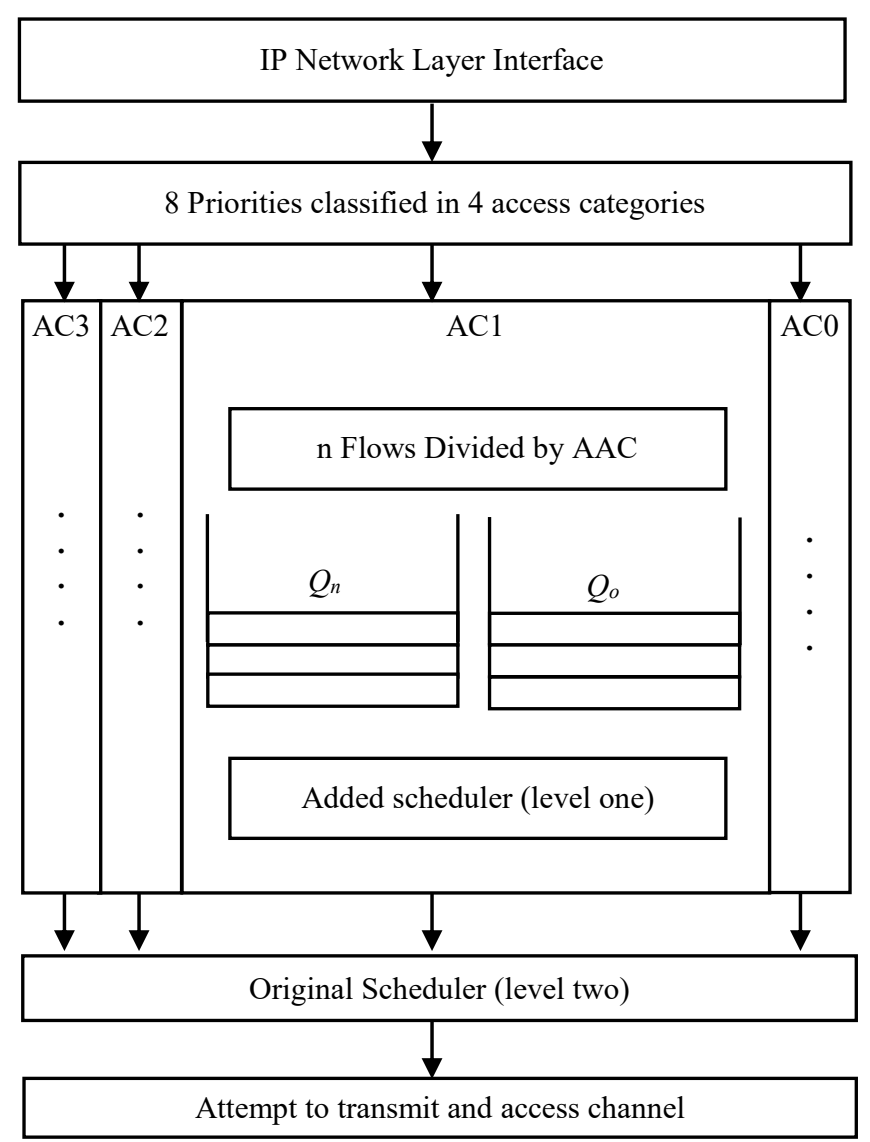

Fig.1(b). Hierarchical Scheduler for 802.11aa/ax using EDCA reference model

Weighted Fair Queuing (WFQ) allocates a portion of the output bandwidth corresponding to the relative weight of each traffic class during periods of congestion. Weight is relevant for WFQ; weights are assigned to each queue. The weight implies the assigned bandwidth for the queue. A higher weight signifies larger assigned bandwidth and smaller delays. If a queue is configured as a low latency queue the weight attribute of this queue is not used and the WFQ scheduler will ignore the value. The Table. 1 and Table. 2 shows the profile configuration and QoS configuration based on ToS classes for WFQ. The maximum number of packets per queue attribute used when the IP interface is congested. This means when the total number of buffered packets in all the queues is reached.

\section{CONFIGURATION}

The network consists of four pairs of video clients. Each pair works on the unique ToS (Type of Service) for data transfer. The connection between the two routers is a likely bottleneck. WFQ queuing can be allowed on each interface in superior routers. Queuing profile and queuing processing mechanism are set in a sub-attribute called Interface Information in IP QoS parameters compound attribute. Classification scheme and number of queues defined in global queuing profiles. Global queuing profiles are defined in the QoS configuration object. 


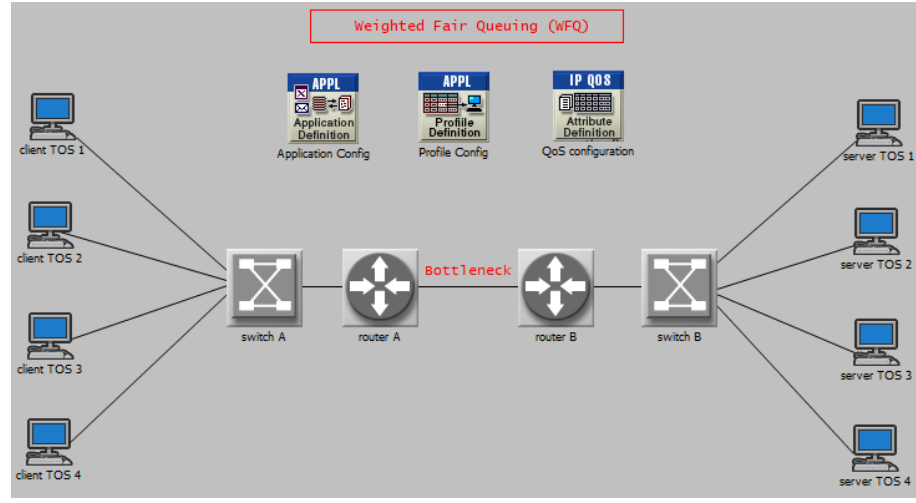

Fig.2. Network Topology

The Fig.2 shows network topology used for DFS scenarios. Each type of service through multiple queues with the help of superior routers. Queue 4 receives ToS 4 traffic, queue 3 receives ToS 3 traffic, queue 2 receives ToS 2 traffic and queue 1 receives ToS 1 traffic. Queues are serviced using Weighted Fair Queuing mechanism. The network configuration is just like that of the previous scenario (WFQ). The only difference is in the WFQ profile details settings where Queue 1 (Q1) is configured to be a Low Latency Queue (LLQ). The LLQ is a stringent priority queue functioning within the normal Weighted Fair Queuing scheduling environment. The item will get better preference within the other queues so that no other queue in the system can be serviced except if the LLQ is empty. The Weight attribute is not useful for the LLQ, and its value becomes ignored by the scheduler. If the LLQ is empty, other queues are serviced based on the standard weighted fair queuing mechanism according to their weight attribute settings.

\section{QOS RELATED STATISTICS}

Simulation offers various statistics specifically relevant to the performance assessment of QoS mechanisms for high efficiency wireless (HEW). All QoS different statistics recognized by OPNET are Node statistics found within the IP Interface category. Most of these statistics are noted for each interface individually. If an interface deploys a QoS mechanism that splits the physical buffer into many logical queues (e. g., WFQ or PQ or CQ) after that these statistics are noted for each logical queue individually [13].

Packet End-to-End Delay (sec): This statistic measures the time elapsed between packets is sent out by a video called party to the time the packet reaches a video calling party. This statistic is collected on a caller basis. In Fig. 3 shows the ETE delay for FIFO scenario, here we noted that queues are almost all full and we cannot differentiate the service it seems to be same for all types of data. Fig.4 shows the ETE delay for WFQ scenario, because of this classification traffic with higher TOS gets better delay. Queue 3 and 4 get their share but let other queues starving of bandwidth. The Fig.7 shows Queue 1 which is designed to become an LLQ, offers the top priority thereby the most significant share of the bandwidth and lowest end-to-end delay.

WFQ Buffer Usage (packets): This statistic measures queue size of each queue on each interface in packets is shown in Fig.6 and Fig.9 for different cases. In Fig.6, traffic is queued in router
A because of the bottleneck. In this example, the WFQ mechanism differentiates traffic between queues based on the type of service (ToS). Queues send traffic proportionally to their weight. In this example, queues with high index have higher weight having less buffer usage. In Fig. $9, Q_{1}$, which is configured to be a LLQ, gets the top priority and thus the highest percentage of the bandwidth and less buffer usage. While Queue 4 which has the highest weight among the other queues gets a full share of the bandwidth, Queues 3 and 2 get starved and have higher delays when compare the results with the WFQ scenario.

WFQ traffic dropped (bits/sec): This statistic measures traffic dropped in bits/sec per queue for each interface. The number of packets dropped can be modified by changing the individual queue size or (for WFQ only) the maximum number of buffered packets per interface.

WFQ traffic received (bits/sec): This statistic measures outgoing traffic received from the central IP CPU of the node (in bits/sec) by each queue per interface. Note that this statistic can be collected only when IP QoS is enabled.

WFQ traffic sent (bits/sec): This statistic records traffic sent to the network (in bits/sec) by each queue per interface. The traffic sent depends on the weight (WFQ), the byte count (custom queuing) or the queue priority (priority queuing) of the queue and the data rate of the link. In Fig.5, clearly highlights the significance of weight in WFQ scenario because which queue has more weight sent higher traffic when compared others where as in Fig.8, $Q_{1}$ assigned as LLQ so irrespective weight $Q_{1}$ must send higher traffic then $Q_{4}, Q_{3}$ and $Q_{2}$.

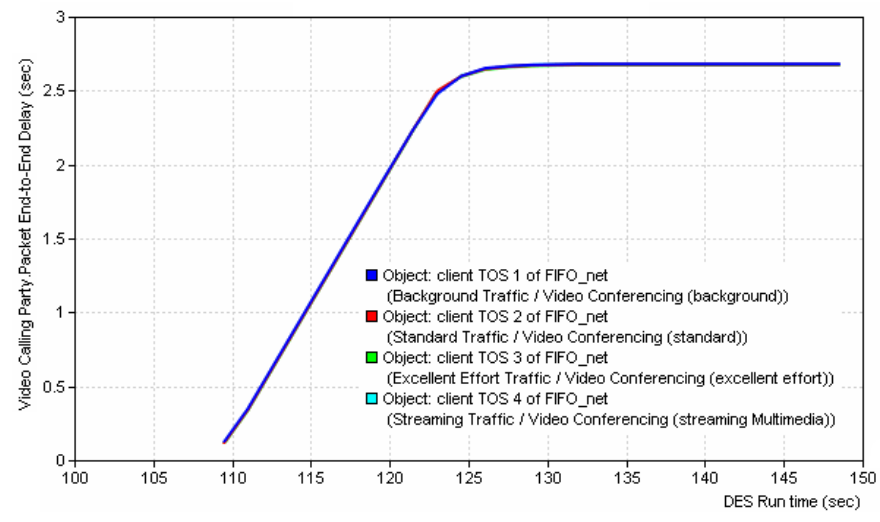

Fig.3. Packet End-to-End Delay using FIFO configuration

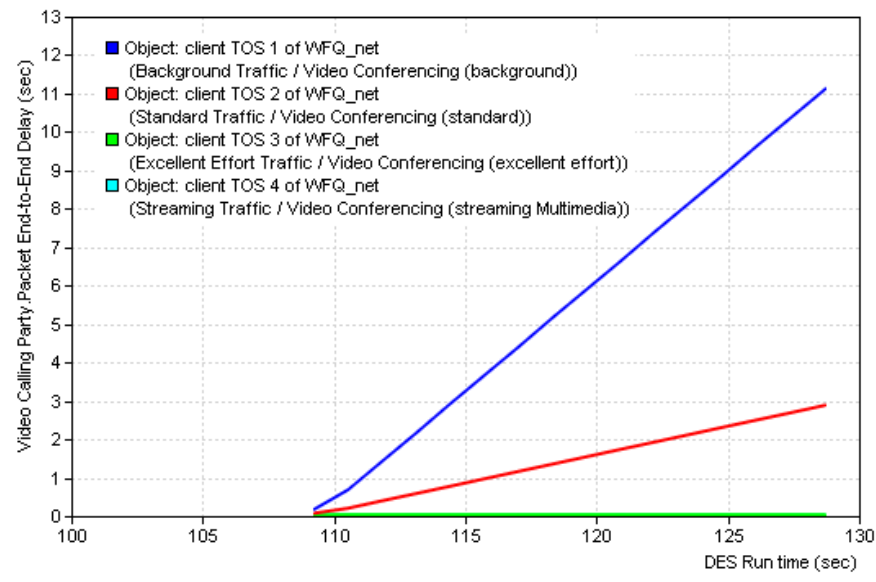

Fig.4. Packet End-to-End Delay using WFQ configuration 
Table.1. Profile Configuration - ToS Based

\begin{tabular}{|c|c|c|}
\hline Profile Name & Applications & Operation Mode \\
\hline $\begin{array}{c}\text { Background } \\
\text { Traffic }\end{array}$ & $\begin{array}{c}\text { Video } \\
\text { Conferencing }\end{array}$ & Simultaneous \\
\hline Standard Traffic & $\begin{array}{c}\text { Video } \\
\text { Conferencing }\end{array}$ & Simultaneous \\
\hline $\begin{array}{c}\text { Excellent Effort } \\
\text { Traffic }\end{array}$ & $\begin{array}{c}\text { Video } \\
\text { Conferencing }\end{array}$ & Simultaneous \\
\hline Streaming Traffic & $\begin{array}{c}\text { Video } \\
\text { Conferencing }\end{array}$ & Simultaneous \\
\hline
\end{tabular}

As the optimum number of buffered packets for each interface is not reached, a queue can easily store packets, no matter what how big the queue. As soon as this specific value is attained, the interface is in a situation of congestion. Because of this, a queue containing more packets compared to its optimum queue size will drop newly arriving packets till either optimum number of packets or optimum queue size falls.

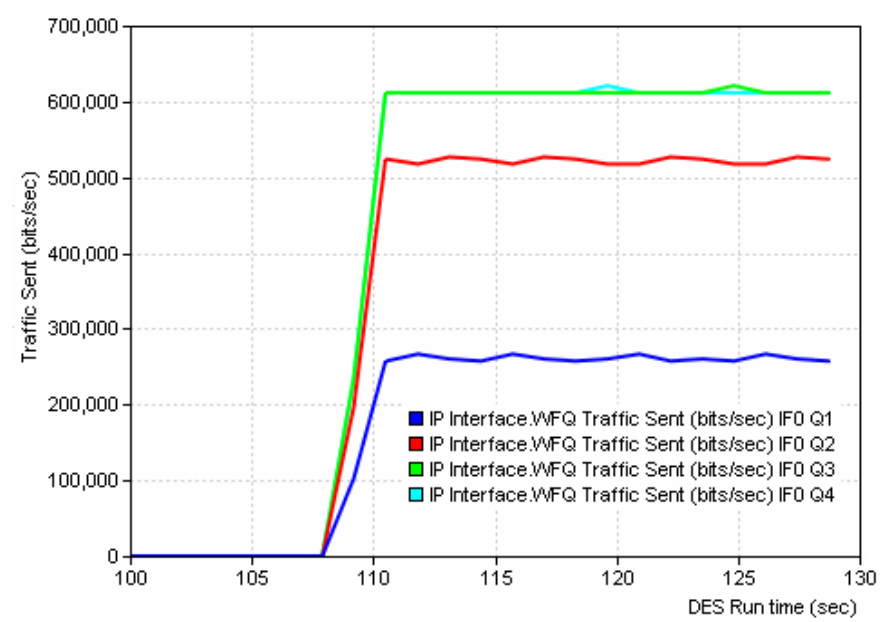

Fig.5. Traffic sent (bits/sec) using WFQ configuration

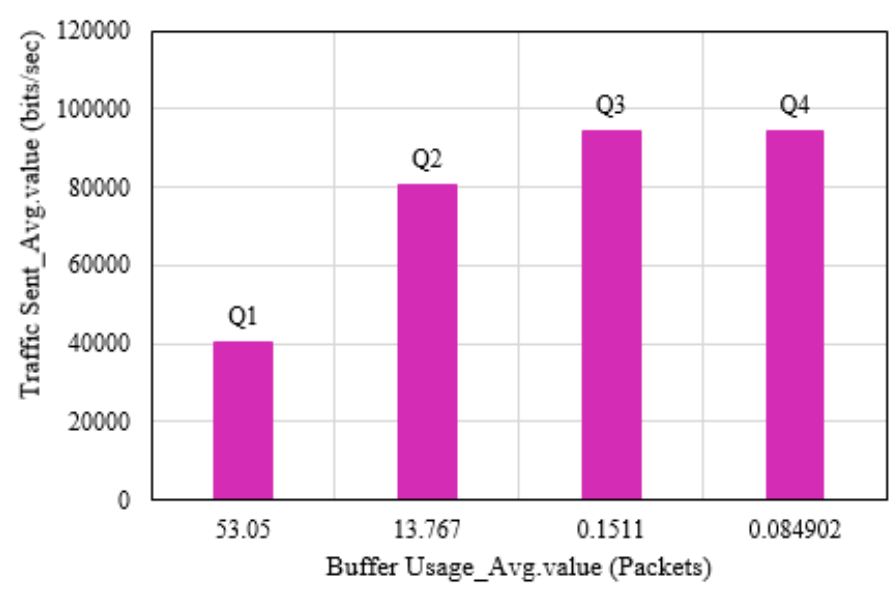

Fig.6. Chart representation using WFQ configured router A

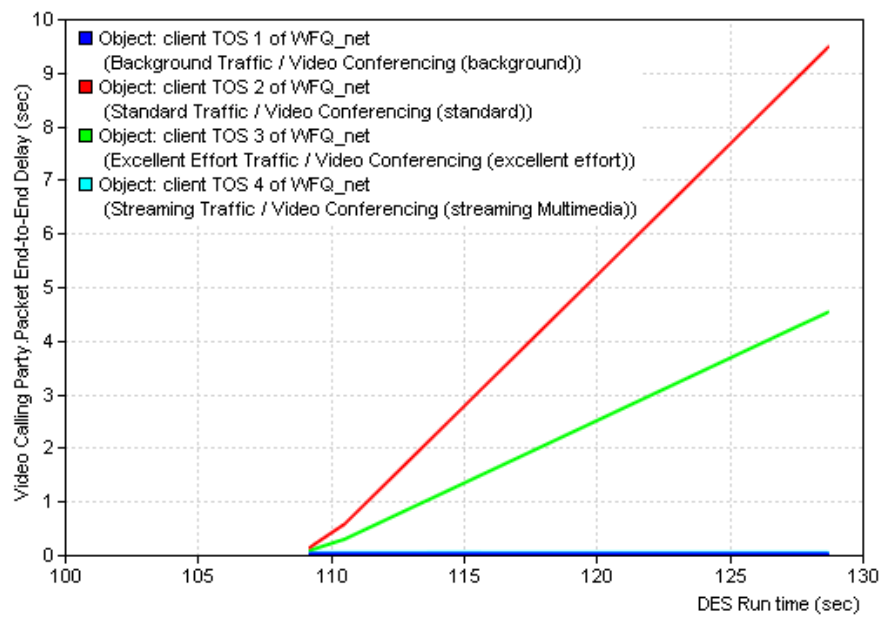

Fig.7. Packet End-to-End Delay using WFQ_LLQ configuration

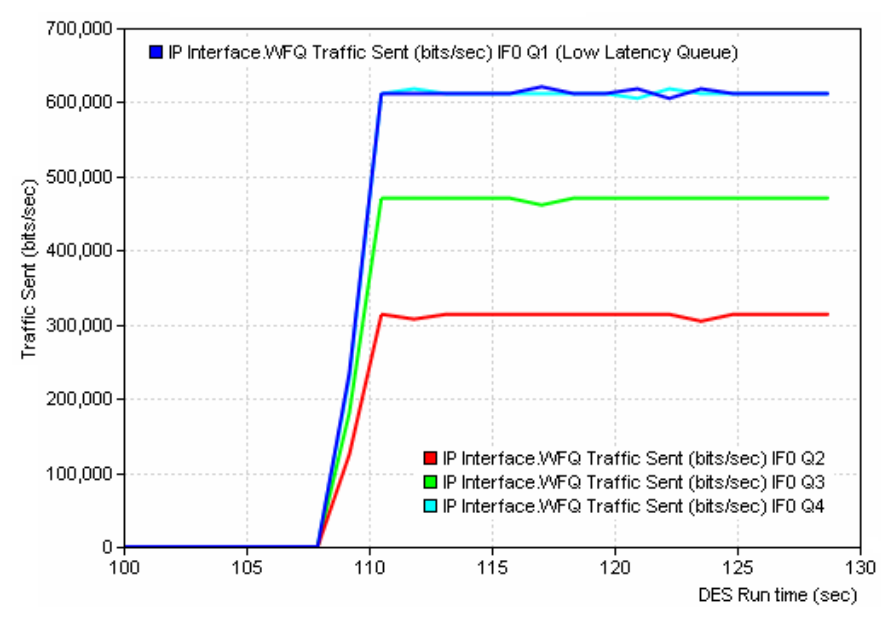

Fig.8. Traffic sent (bits/sec) using WFQ_LLQ configuration

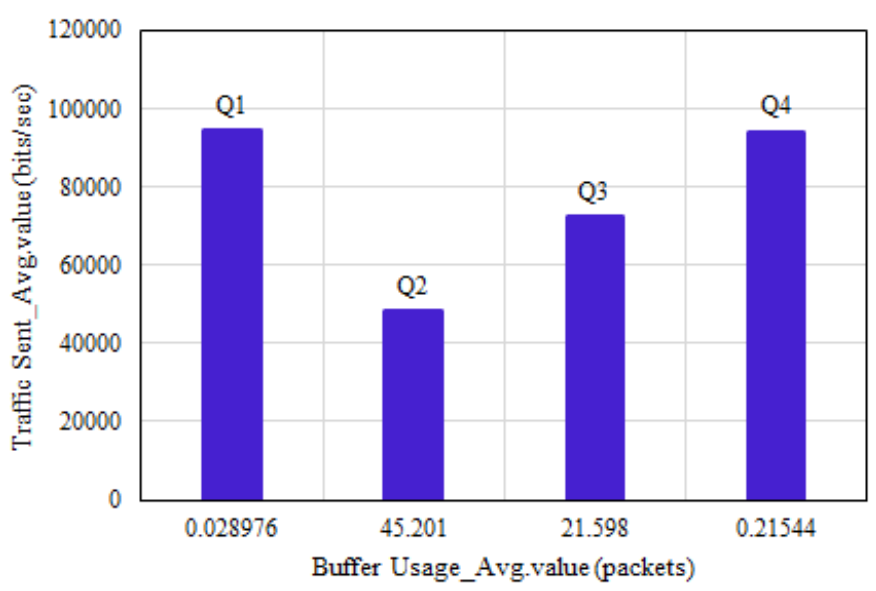

Fig.9. Chart representation using WFQ_LLQ configuration router $\mathrm{A}$ 
Table.2. QoS configuration in WFQ profiles - ToS Based

\begin{tabular}{|c|c|c|c|}
\hline Weight & $\begin{array}{c}\text { Maximum } \\
\text { Queue Size } \\
\text { (pkts) }\end{array}$ & $\begin{array}{c}\text { Classification } \\
\text { scheme }\end{array}$ & $\begin{array}{c}\text { Queue } \\
\text { Category }\end{array}$ \\
\hline 1.0 & 500 & Best Effort $\left(Q_{0}\right)$ & Default Queue \\
\hline 10 & 500 & Background $\left(Q_{1}\right)$ & LLQ \\
\hline 20 & 500 & Standard $\left(Q_{2}\right)$ & None \\
\hline 30 & 500 & $\begin{array}{c}\text { Excellent Effort } \\
\left(Q_{3}\right)\end{array}$ & None \\
\hline 40 & 500 & $\begin{array}{c}\text { Streaming } \\
\text { Multimedia }\left(Q_{4}\right)\end{array}$ & None \\
\hline 50 & 500 & $\begin{array}{c}\text { Interactive } \\
\text { Multimedia }\left(Q_{5}\right)\end{array}$ & None \\
\hline 60 & 500 & $\begin{array}{c}\text { Interactive Voice } \\
\left(Q_{6}\right)\end{array}$ & None \\
\hline 70 & 500 & Reserved $\left(Q_{7}\right)$ & None \\
\hline
\end{tabular}

\section{MULTI-USER MODE OF EDCA - 802.11AX}

In this section, we discussed about the channel access for trigger frame and its new set of parameters related to uplink transmission. Virtual queue for $U L M U$ transmission to remove the worries regarding $U L M U$ discussed.

\subsection{CHANNEL ACCESS FOR TRIGGER FRAME}

$M U U L$ begins with Trigger Frame transmitted by $A P$; we need to define a procedure whether to transmit $D L$ data or to transmit trigger frame and hard to know which EDCA parameters are appropriate for $U L$ transmission (see Fig.10). When $A C_{M U}$ wins the virtual contention, trigger frame is transmitted with trigger frame access category $\left(A C_{M U}\right)$ EDCA parameters (see Fig.11). EDCA parameters like minimum Backoff $(B O)$, maximum $B O(=C W)$, TXOP, and AIFSN for $A C_{M U}$ needs to be defined; not only fixed value but also the variable value which is based on queue length could be considered (Legacy ACs could be reused for $A C_{M U}$, e.g., $A C_{V I}$ ). MU-RTS may follow EDCA of the following channel access procedure of $M U$ data transmission. $U L$ $M U$ for management frames including BSR procedure may use different mechanism and $U L M U$ parameters [6] [12].

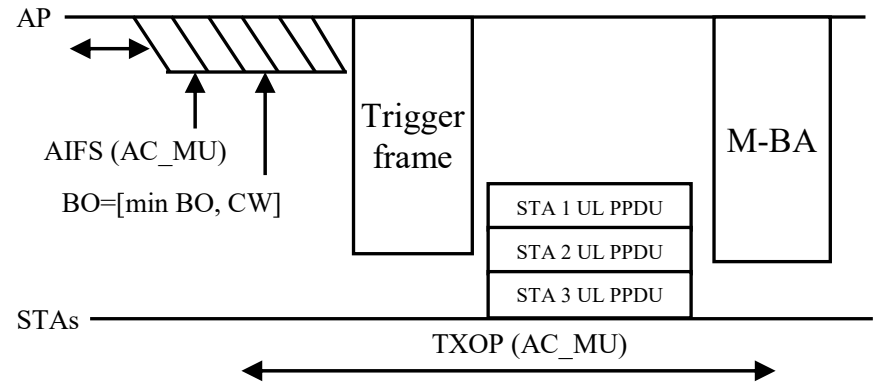

Fig.10. Channel Access for Trigger Frame

Buffer Status Report (BSR) procedure adopted for 802.11ax $M U U L$, and it should be used for $A P$ to determine $U L$ transmission scheduling, i.e., $D L / U L$ transmission and UL Trigger Frame transmission timing. Although Buffer Status
Report procedure is defined, Access Category or TID of $U L$ data cannot be regulated for $M U U L$ transmission. Trigger Frame cannot restrict transmission of any AC or TID of STAs Therefore, applying for legacy access category scheme including sharing a TXOP scheme for Trigger Frame Channel Access is not feasible [1] [7] [8].

Table.3. Example of UL MU Contention Control

\begin{tabular}{|c|c|c|c|}
\hline Category & $\begin{array}{c}\text { Queue Length } \\
(\boldsymbol{Q})\end{array}$ & Min $\boldsymbol{B O}$ & $\begin{array}{c}\boldsymbol{C W} \\
(\operatorname{Max} \boldsymbol{B O})\end{array}$ \\
\hline 1 (Hold $B O)$ & $Q<T h \_1$ & 1024 (Hold) & 1024 (Hold) \\
\hline 2 & $T h_{1} \leq Q<T h_{2}$ & 8 & 31 \\
\hline 3 & $T h_{2} \leq Q<T h_{3}$ & 0 & 31 \\
\hline 4 & $\vdots$ & $\vdots$ & $\vdots$ \\
\hline$N+1(N \geq 1)$ & $T h_{n}<Q$ & 0 & 0 \\
\hline
\end{tabular}

$U L M U$ Access Category and $U L M U$ virtual queue can clarify the two ambiguities of $U L M U$ channel Access: (1) when or how often trigger frames should be transmitted and (2) which EDCA parameters should Trigger Frame follow. The Queue size of $A C_{M U}$ is determined with cumulated Buffer Status Report information and STAs $S U$ transmission status. For internal contention, $U L M U$ virtual contention parameters controlled by virtual queue size. Large virtual queue size should provide a high probability of channel access requires small Backoff $(B O)$ value.

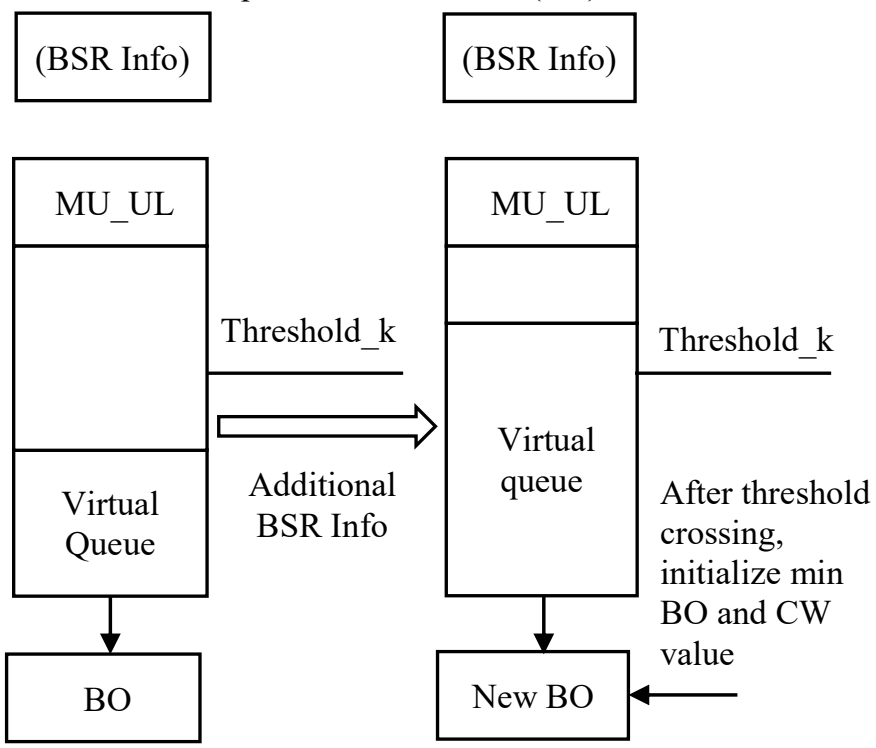

Fig.11. BSR adopted in UL MU AC for Trigger Frame

If $A C_{M U}$ wins the internal contention, trigger frame follows the channel access parameters of $A C_{M U}$. Backoff $(B O)$ values should be controlled depending on the $A C_{M U}$ queue size, and $B O$ values should be monitored with contention window $(C W)$ size. Unlike the conventional $B O$, minimum (Min) $B O$ should be regulated to solicit many STAs. $A C_{M U}$ gets virtual back-off values in the range of [Min $B O, C W]$ instead of $[0, C W]$. If the queue length of $A C_{M U}(U L)$ is smaller than the specific threshold value, then trigger frame cannot get a transmission opportunity due to its large minimum backoff number. Either fixed $C W$ or variable $C W$ can be used. $N>1$ : variable $C W ; N=1$ : fixed $C W$ with $U L M U$ threshold. 


\section{NEW EDCA SET FOR MU OPPORTUNITY}

$M U$ EDCA set is a new EDCA parameter set that it lowers STAs channel access opportunity and could be used for enhancing 11 ax efficiency. When $M U$ EDCA is indicated $H E$ non-AP STAs shall use $M U$ EDCA parameters for its SU channel access. $M U$ EDCA indication might be performed by Trigger Frame for some HE STAs or all the $H E$ STAs, and for each $A C$ or all $A C s$, and $S U$ EDCA indication is possible, also (Return to conventional EDCA). Because of $M U$ EDCA set, STAs would transmit $H E$ trigger based PPDUs rather than another $S U$ PPDUs.

$M U$ EDCA parameters for the MSDU can be applied when $U L$ MSDU is arrived after EDCA mode indication changed by Trigger Frame. This will not create any issues for $M U$ transmission which is illustrate in Fig.10 and Fig.11. Retransmission count affect its new back-off procedure of $M U$ EDCA is shown in Fig.12. If retransmission occurred, how new EDCA $C W$ would be applied to the existing channel access procedure use $C W_{\text {min }}$ mu or larger one or doubled $C W$ of SU EDCA parameter. Like MU Mode initiation, procedure after $M U$ MODE expiration need to be considered. After MU Mode truncation or $M U$ EDCA timer expiration, procedures for the existing back-off timer and retransmission timer need to be considered as well as its MU Mode initiation procedure [8] [15]-[16].

\subsection{ISSUE ON THE $C W_{\max }$ VALUE IN MU EDCA}

$C W_{\max }$ values in MU and Legacy modes: When an HE STA switches from MU mode to Legacy mode, its $C W$ value may exceed the legacy $C W_{\max }[A C]$ value. MU EDCA parameter set is proposed to lower channel access probability of HE STAs, $C W_{\max }$ value of MU set is likely to be greater than that of Legacy set shown in Fig.12 [17]. If the STA experiences (internal) collision, it shall follow the procedure defined in $T G_{a x}$ draft EDCA backoff procedure. Proposed fix: If the STA experiences another collision, it follows the EDCA backoff procedure. When the current $\mathrm{CW}$ value exceeds the $C W_{\max }[A C]$, there is no defined rule to refer. Therefore, we propose to modify the $\mathrm{CW}$ value resetting rule in case the current $\mathrm{CW}$ value already exceeds $\mathrm{CWmax}$. For an $\mathrm{HE}$ STA, if $C W[A C]>C W_{\max }[A C], C W[A C]$ shall be set to $C W_{\max }[A C]$.
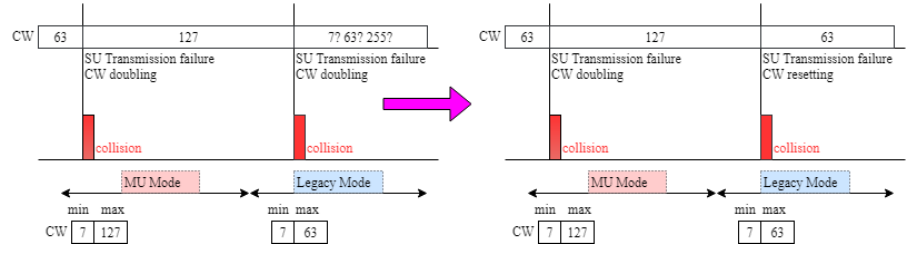

Fig.12. $C W_{\max }$ adjustments for MU EDCA

\subsection{POSSIBLE SOLUTIONS FOR MU MODE EDCA}

Option 1: Reset back-off and CW doubling i.e., reset its backoff number based on a changed EDCA set and retransmission history is not considered ( $C W_{\text {min_mu }}$ for ReTx). Reset back-off but $C W$ based on number of ReTx i.e., Reset its back-off number based on a changed EDCA set. Using $C W$ value considering retransmission is $2^{(n-1)} \times C W_{\text {min }}$ mu.

Option 2: Sustain last EDCA procedure until transmission success or retransmission limit ( $M U$ mode is applied after EDCA procedure of last frame). Sustain last EDCA procedure only once whether the transmission is success or not, next transmission follows option 1 (Do not reset back-off number but applying new CW immediately)

\section{CONCLUSION}

IEEE $802.11 \mathrm{ax}$ is the sixth generation of Wi-Fi because of new multiple access schemes to deal with a different level of service to various traffic flows with the help of well-known distributed weighted fair queuing traffic scheduler. $M U U L$ virtual queue based on BSR information could be a solution for $11 \mathrm{ax}$ EDCA also we verified through OPNET simulation. Variable virtual contention parameters for $A C_{M U}$ could control when and how often trigger frames are transmitted. $M U$ mode EDCA set is a new EDCA parameter set that is $5^{\text {th }}$ percentile STAs channel access opportunity and could be used for enhancing $11 \mathrm{ax}$ efficiency. Regardless of $U L$ PPDU contents, Trigger frame always needs to be transmitted following $A C_{M U}$. Addressing an issue regarding $\mathrm{CW}$ value setting after an STA switches back to the Legacy mode from $M U$ mode.

\section{REFERENCES}

[1] D.J. Deng, S.Y. Lien, J. Lee and K.C. Chen, "On Qualityof-Service Provisioning in IEEE 802.11ax WLANs", IEEE Access, Vol. 4, pp. 6086-6104, 2016.

[2] Hui Zhao et.al, "IEEE 802.11-13/1077r1: EDCA Enhancements for HEW", Available at: http://www.ieee802.org/11/Reports/hew_update.htm.

[3] Eldad Perahia and Robert Stacey, "Next Generation Wireless LANs: 802.11n and 802.11ac", $2^{\text {nd }}$ Edition, Cambridge University Press, 2013, Ch. 8 and 10.

[4] S. Mangold et al., "Analysis of IEEE 802.11e for QoS Support in Wireless LANs", IEEE Wireless Communications, Vol. 10, No. 6, pp. 40-50, 2003.

[5] D. J. Deng et al., "IEEE 802.11ax: Highly Efficient WLANs for Intelligent Information Infrastructure", IEEE Communications Magazine, Vol. 55, No. 12, pp. 52-59, 2017.

[6] K. Kosek-Szott, M. Natkaniec and L. Prasnal, "IEEE 802.11aa Intra-AC Prioritization - A New method of Increasing the Granularity of Traffic Prioritization in WLANs", Proceedings of the IEEE Symposium on Computers and Communications, pp. 1-6, 2014.

[7] A. Boukerche, "Handbook of Algorithms for Wireless Networking and Mobile Computing”, CRC Press, 2005.

[8] IEEEP 802.11 Task Group AX Reports Update, Available at: http://www.ieee802.org/11/Reports/tgax_update.htm

[9] IEEE Standard for Information TechnologyTelecommunications and Information Exchange between Systems Local and Metropolitan Area Networks-Specific Requirements-Part 11: Wireless LAN Medium Access Control (MAC) and Physical Layer (PHY) Specifications, IEEE Std 802.11-2016 (Revision of IEEE Std 802.11-2012), 2016.

[10] B. Bellalta, "IEEE 802.11ax: High-efficiency WLANs", IEEE Wireless Communications, Vol. 23, No. 1, pp. 38-46, 2016. 
[11] F.M. Abinader, S. Choudhury and V.A. De souse, "Distributed Wi-Fi Interference Coordination for Dense Deployments", Wireless Personal Communications, Vol. 97, No. 1, pp. 1033-105, 2017.

[12] E. Khorov, V. Loginov and A. Lyakhov, "Several EDCA Parameter Sets for Improving Channel Access in IEEE 802.11ax Networks", Proceedings of International Symposium on Wireless Communication Systems, pp. 419423, 2016.

[13] Adarshpal S Sethi and Vasil Y Hnatyshin, "The Practical OPNET User Guide for Computer Network Simulation", CRC Press, 2012.

[14] B.T Vijay and B Malarkodi, "A Study of IEEE 802.11aa", Proceedings of $8^{\text {th }}$ International Conference on Computing,
Communication and Networking Technologies, pp. 1-6, 2017.

[15] H. Abuzanat, B. Trouillet and A. Toguyeni, "FQ-EDCA: An Extension of EDCA to Improve Fairness in Ad-Hoc Wireless Network", Proceedings of International Conference on Computers and Industrial Engineering, pp. 1617-1622, 2009.

[16] B. Rebekka, S. Sudheep and B. Malarkodi, "Priority-Based Quality of Service Guaranteed Radio Resource Allocation in Long Term Evolution Network", International Journal of Information and Communication Technology, Vol. 9, No. 4, pp. 420-437, 2015. 